\title{
A GEOMETRIC ISOMORPHISM WITH APPLICATIONS TO CLOSED LOOP CONTROLS
}

\author{
Robert B. Gardner ${ }^{1}$ \\ William F. SHADWICK ${ }^{2}$ \\ George R. WiLKEnS ${ }^{3}$
}

\begin{abstract}
Feedback equivalence of $n$ state, $n-1$ control systems divides such systems into two invariant classes. We show that class 1 corresponds, via a geometric isomorphism, to classical Lagrangian variational problems. We prove the existence of time critical closed loop controls for systems which satisfy the non-degeneracy condition that the analogue of the Hessian for the Lagrangian problem have full rank. We show that the vanishing of this Hessian characterizes the affine linear systems in class 1 and identify the rank condition for local controllabilty for such systems as the non-vanishing of a differential invariant. The affine linear systems in class 2 are also characterized by the vanishing of an invariant and the rank condition is identified.
\end{abstract}

AMS(MOS) subject classifications. 49, 53

\section{INTRODUCTION}

In this paper we consider the problem of feedback equivalence of control systems, with $n$ states and $n-1$ controls, as the equivalence problem for systems

$$
\frac{d x}{d t}=F(x, u) \quad x \in \mathbf{R}^{n}, \quad u \in \mathbf{R}^{n-1},
$$

under diffeomorphisms of the form

$$
\Phi(t, x, u)=(t, \phi(x), \psi(x, u)) .
$$

By making use of Cartan's method of equivalence $[\mathbf{3}],[\mathbf{5}],[\mathbf{6}]$ we obtain an invariant splitting of such systems into two classes. The first of these, on which we focus our attention, is identified via a geometric isomorphism, with classical single integral variational problems. The existence of this isomorphism means that all of the rich geometry of classical Lagrangian mechanics is encoded in the control system (1.1) and may be applied to its study. The most basic elements of the Lagrangian problem are the notions of regularity and of the Euler-Lagrange equations for critical curves. We show that, as one would hope, these concepts translate into basic features of the control problem. The vanishing of the Hessian for the associated Lagrangian is necessary and sufficient for the control system to be equivalent to one in affine-linear form

$$
\frac{d x}{d t}=f(x)+\sum g_{i}(x) u^{i}
$$

At the other extreme, when the Hessian has full rank, we show that the Euler-Lagrange equations may be solved to provide closed loop controls. As the Lagrangian functional, applied to solution curves of (1.1), measures the time from initial to final endpoints, these controls are time critical. Moreover, for a certain subclass of control systems, they are the geodesics of a pseudo-Riemannian metric intrinsic to the system as Wilkens found in the case $n=3$. [13].

\footnotetext{
${ }^{1}$ Mathematics Department, University of North Carolina, Chapel Hill, NC 27599-3250. Supported by NSF Grant number DMS8505434.

${ }^{2}$ Mathematics Department, Duke University, Durham, NC 27706. Permanent Address: Pure Mathematics Department, University of Waterloo, Waterloo, Ontario N2L 3G1. Supported by NSERC Grant number A7895.

${ }^{3}$ Mathematics Department, North Carolina State University, Raleigh, NC 27695-8205.
} 
The second class of systems corresponds to a non-classical variational problem of the sort studied by Griffiths [8]. We will pursue the study of this aspect of the problem elsewhere. We show that every system in this class can be put in the form

$$
\begin{aligned}
\frac{d x^{i}}{d t} & =u^{i} \quad 1 \leq i \leq n-1 \\
\frac{d x^{n}}{d t} & =g(x, u)
\end{aligned}
$$

where $g$ is homogeneous of degree 1 in the controls. We give necessary and sufficient conditions for the system to be affine linear, in which case $g(x, u)=\sum g_{i}(x) u^{i}$. For such systems the controllability condition $[\mathbf{1}],[\mathbf{1 1}]$ is that an invariant should not vanish. As there is no drift term, the condition will fail for any linearizable system in this class, however there is an invariant skew symmetric matrix $A$ which determines at least rank $A$ equivalence classes of controllable affine linear systems.

Finally we note that while the restriction to consideration of systems with $n$ states and $n-1$ controls is essential to the identification of a classical first order variational problem, the same techniques apply to the case of $n$ states and $p$ controls. This case requires the analysis of more general variational problems and is currently being studied.

\section{The Equivalence Problem}

Given the system (1.1) on $U_{0} \subset \mathbf{R}^{2 n}$ with coordinates $t, x^{1}, \ldots, x^{n}, u^{1}, \ldots, u^{n-1}$ and a second system

$$
\frac{d \bar{x}}{d \bar{t}}=\bar{f}(\bar{x}, \bar{u})
$$

on $\bar{U}_{0} \subset \mathbf{R}^{2 n}$, the problem of local equivalence under feedback is the equivalence problem for maps

$$
\Phi: U_{0} \rightarrow \bar{U}_{0}
$$

of the form

$$
\begin{aligned}
& \bar{t} \circ \Phi=t \\
& \bar{x} \circ \Phi=\phi(x) \\
& \bar{u} \circ \Phi=\psi(x, u)
\end{aligned}
$$

which satisfy

$$
\Phi^{*}(d \bar{x}-\bar{f} d \bar{t})=A(d x-f d t)
$$

for some $A \in G L(n, \mathbf{R})$. This is an overdetermined equivalence problem and leads, as discussed in $[\mathbf{7}]$, to the following problem in standard form.

Let $U$ and $\bar{U}$ be open sets on which $f$ and $\bar{f}$ are non-zero and let $A_{0}$ and $\bar{A}_{0}$ be maps from $U$ and $\bar{U}$ to $G L(n, \mathbf{R})$ such that

$$
A_{0} f=\bar{A}_{0} \bar{f}={ }^{\mathrm{t}}(1,0, \ldots, 0) .
$$

A diffeomorphism $\Phi$ from $U$ to $\bar{U}$ satisfies (2.1) and (2.2) if and only if

$$
\bar{t} \circ \Phi=t
$$

and

$$
\Phi^{*}\left(\begin{array}{c}
\bar{A}_{0} d \bar{x} \\
d \bar{u}
\end{array}\right)=\left(\begin{array}{ccc}
1 & A & 0 \\
0 & B & 0 \\
C & D & E
\end{array}\right)\left(\begin{array}{c}
A_{0} d x \\
d u
\end{array}\right)
$$


with $B, E \in G L(n-1, \mathbf{R})$. This now has the form considered in [5], [6] where $G$ is the subgroup of $G L(2 n-1, \mathbf{R})$ of matrices of the form

$$
\left(\begin{array}{lll}
1 & A & 0 \\
0 & B & 0 \\
C & D & E
\end{array}\right)
$$

with $B$ and $E$ in $G L(n-1, \mathbf{R})$. Thus we construct the vector of 1 -forms ${ }^{\mathrm{t}}\left(\eta^{1}, \ldots, \eta^{n}, \mu^{1}, \ldots, \mu^{n-1}\right)$ on $U \times G$ given by

$$
\left(\begin{array}{c}
\eta \\
\mu
\end{array}\right)=\left(\begin{array}{ccc}
1 & A & 0 \\
0 & B & 0 \\
C & D & E
\end{array}\right)\left(\begin{array}{c}
\eta_{U} \\
\mu_{U}
\end{array}\right)
$$

where

$$
\left(\begin{array}{c}
\eta_{U} \\
\mu_{U}
\end{array}\right)=\left(\begin{array}{c}
A_{0} d x \\
d u
\end{array}\right)
$$

We now turn to the equivalence of classical first order Lagrangian problems and show that it leads to the same structure. Here we consider functionals

$$
\mathcal{L}(c)=\int_{c} L(\tau, q, \dot{q}) d \tau
$$

over curves $c$ in $\mathbf{R}^{2 p+1}$ which satisfy

$$
\dot{q}^{i} \circ c=\frac{d}{d \tau}\left(q^{i} \circ c\right) \quad 1 \leq i \leq p .
$$

These curves are integrals of the contact system

$$
\theta^{i}:=d q^{i}-\dot{q}^{i} d \tau, \quad 1 \leq i \leq p
$$

with independence condition $d \tau \neq 0$. Given a second functional $\overline{\mathcal{L}}$, we will say that $\mathcal{L}$ and $\overline{\mathcal{L}}$ are equivalent if there is a contact transformation $\Phi$ such that

$$
\Phi^{*} \bar{L} d \bar{\tau} \equiv L d \tau \quad\left(\bmod \theta^{i}\right)
$$

This is clearly an equivalence relation and preserves the value of the functionals on integrals of the contact system.

If we complete $\{L d \tau, \theta\}$ and $\{\bar{L} d \bar{\tau}, \bar{\theta}\}$ to coframes by adding 1 -forms $\zeta$ and $\bar{\zeta}$ and use the fact that $\Phi$ must preserve the contact system $\left\{\theta^{i}\right\}$, we may summarize the conditions on the Jacobian of an equivalence $\Phi$ by

$$
\Phi^{*}\left(\begin{array}{c}
\bar{L} d \bar{\tau} \\
\bar{\theta} \\
\bar{\zeta}
\end{array}\right)=\left(\begin{array}{ccc}
1 & A & 0 \\
0 & B & 0 \\
C & D & E
\end{array}\right)\left(\begin{array}{c}
L d \tau \\
\theta \\
\zeta
\end{array}\right)
$$

Thus, if $p=n-1,(2.6)$ and (2.4) suggest the identification on $\eta_{U}^{1}$ with $L d \tau$ and $\left\{\eta_{U}^{2}, \ldots, \eta_{U}^{n}\right\}$ with the contact system. It is easy to verify that if $c$ is a solution of (1.1) we have

$$
c^{*} A_{0} d x={ }^{\mathrm{t}}(d t, 0, \ldots, 0)
$$

so $c^{*} \eta^{1}=d t, c^{*} \eta^{i}=0,2 \leq i \leq n$ and

$$
\int_{c} \eta^{1}=\int_{t_{0}}^{t_{1}} d t
$$


Thus the variational problem which we have identified is the time optimization problem for solutions of (1.1).

There is an integrability condition which obstructs the identification of $\left\{\eta^{2}, \ldots, \eta^{n}\right\}$ with a contact system: the derived structure of $\eta^{2}, \ldots, \eta^{n}$ must coincide with that of $\theta^{1}, \ldots, \theta^{n-1}$. We now show how this invariant arises by pursuing the equivalence problem calculation.

For the system $(2.4)$ with $\bar{\eta}={ }^{\mathrm{t}}\left(\eta^{2}, \ldots, \eta^{n}\right)$, the structure equations take the form

$$
\begin{aligned}
d \eta^{1} & ={ }^{\mathrm{t}} \alpha \wedge \bar{\eta}+\eta^{1 \mathrm{t}} m \wedge \mu \\
d \bar{\eta} & =\beta \wedge \bar{\eta}+\eta^{1} M \wedge \mu \\
d \mu & =\gamma \wedge \eta^{1}+\delta \wedge \bar{\eta}+\varepsilon \wedge \mu
\end{aligned}
$$

after all torsion has been absorbed.

The matrix $\mathcal{M}:=\left(\begin{array}{c}{ }^{\mathrm{t}} \\ M\end{array}\right)$ is, up to left and right multiplication, just

$$
A_{0}\left(\frac{\partial f}{\partial u}\right)
$$

and hence the full rank case is the only one in which all $n-1$ controls are actually present. From the infinitesimal action on ${ }^{\mathrm{t}} m$ and $M$,

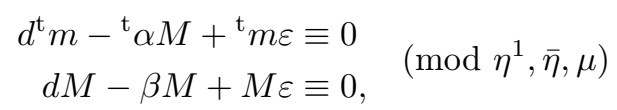

we see that the rank of $M$ is also an invariant and thus there are two cases to consider: rank $M=n-2$, and rank $M=n-1$. In either case, as we may assume that $\operatorname{rank} \mathcal{M}=n-1$, we may put the original control system in a form similar to the ones observed by Hermann [9]

$$
\begin{aligned}
& \frac{d x^{i}}{d t}=u^{i} \quad 1 \leq i \leq n-1 \\
& \frac{d x^{n}}{d t}=g(x, u),
\end{aligned}
$$

and we may choose $A_{0}$ to be given by

$$
A_{0}=\left(\begin{array}{ccccc}
1 / u^{1} & 0 & 0 & \ldots & 0 \\
-u^{2} & u^{1} & 0 & \ldots & 0 \\
\vdots & & \ddots & & \vdots \\
-u^{n-1} & 0 & \ldots & u^{1} & 0 \\
-g & 0 & \ldots & 0 & u^{1}
\end{array}\right)
$$

A short calculation now shows that rank $M=n-2$ if and only if $\hat{g}:=g-\sum u^{i} \partial g / \partial u^{i}=0$, i.e. if and only if $g$ is homogeneous of degree 1 , which is clearly a non-generic condition.

Case 1) Rank $M=n-1$.

We have $\hat{g}:=g-\sum u^{i} \partial g / \partial u^{i} \neq 0$ and from (2.8) we may normalize $M$ to the identity and ${ }^{\mathrm{t}} m$ to 0 to obtain new congruences

$$
\begin{aligned}
{ }^{\mathrm{t}} \bar{\alpha} & \equiv 0 \\
\beta & \equiv \varepsilon
\end{aligned} \quad\left(\bmod \eta^{1}, \bar{\eta}, \mu\right)
$$

In this case, the identification suggested above is actually an isomorphism, as the structure equations are now identical with those of the classical Lagrangian problem [6], and we have the following theorem. 
THEOREM 2.1. If rank $M=n-1$ the system is isomorphic to the classical first order Lagrangian system in $n-1$ dependent variables.

The remainder of the equivalence problem is now precisely the calculations for the Lagrangian case carried out by Bryant and Gardner [6] and the parametric form of the invariants has recently been investigated by Sutton [12]. For our purposes, it suffices to consider the invariants which are introduced by the congruences $\left(2.8^{\prime}\right)$.

After absorption of torsion terms the structure equations become

$$
\begin{aligned}
d \eta^{1} & ={ }^{\mathrm{t}} \mu H \wedge \bar{\eta}+{ }^{\mathrm{t}} \bar{\eta} S \wedge \bar{\eta}+\eta^{1 \mathrm{t}} \bar{v} \wedge \bar{\eta} \\
d \bar{\eta} & =\beta \wedge \bar{\eta}+\eta^{1} \wedge \mu \\
d \mu & =\gamma \wedge \eta^{1}+\delta \wedge \bar{\eta}+\beta \wedge \mu .
\end{aligned}
$$

The integrability condition $d^{2}=0$ shows that $H$ is a symmetric matrix, and in the Lagrangian variables, $H$ is, up to conjugation, just the Hessian matrix $\partial^{2} L / \partial \dot{q}^{i} \partial \dot{q}^{j}$.

Theorem 2.2. If $\hat{g} \neq 0, H$ vanishes if and only if the system (1.1) is equivalent to

$$
\begin{aligned}
\frac{d x^{i}}{d t} & =u^{i} \quad 1 \leq i \leq n-1 \\
\frac{d x^{n}}{d t} & =f(x)+\sum g_{i}(x) u^{i} .
\end{aligned}
$$

If $H=0$ the rank condition for local controllability is satisfied if and only if $d \eta^{1} \neq 0$. The condition $d \eta^{1}=0$ gives a conservation law generalizing Hermes [10].

Proof: To establish this result we look at the explicit parametric calculation for the normalization of ${ }^{\mathrm{t}} m$ to 0 . If we adopt the parametrization given by (2.9) and (2.10) then

$$
\eta^{1}=\frac{d x^{1}}{u^{1}}+A_{1}\left(u^{1} d x^{2}-u^{2} d x^{1}\right)+\cdots+A_{n-2}\left(u^{1} d x^{n-2}-u^{n-2} d x^{1}\right)+A_{n-1}\left(u^{1} d x^{n}-g d x^{1}\right) .
$$

It is easy to check that $d \eta^{1} \equiv 0(\bmod \bar{\eta})$ requires

$$
\begin{gathered}
A_{1}=-A_{n-1} \frac{\partial g}{\partial u^{2}} \\
A_{2}=-A_{n-1} \frac{\partial g}{\partial u^{3}} \\
\vdots \\
A_{n-2}=-A_{n-1} \frac{\partial g}{\partial u^{n-1}}
\end{gathered}
$$

and $1 / u^{1}=A_{n-1} \hat{g}$. As $\hat{g} \neq 0$, we may solve for $A_{n-1}$ to obtain

$$
\eta^{1}=\frac{1}{\hat{g}}\left(d x^{n}-\frac{\partial g}{\partial u^{1}} d x^{1}-\frac{\partial g}{\partial u^{2}} d x^{2}-\cdots-\frac{\partial g}{\partial u^{n-1}} d x^{n-1}\right) .
$$

Now $H=0$ means

$$
d \eta^{1} \wedge \eta^{1}={ }^{\mathrm{t}} \bar{\eta} S \wedge \bar{\eta} \wedge \eta^{1}
$$

which has no component in $\mu$ and hence no component in $d u$. But

$$
\begin{aligned}
& d \eta^{1} \wedge \eta^{1}=-\frac{1}{\hat{g}^{2}}\left\{d\left(\frac{\partial g}{\partial u^{1}}\right) \wedge d x^{1}+\cdots+d\left(\frac{\partial g}{\partial u^{n-1}}\right) \wedge d x^{n-1}\right\} \wedge \\
&\left\{d x^{n}-\frac{\partial g}{\partial u^{1}} d x^{1}-\frac{\partial g}{\partial u^{2}} d x^{2}-\cdots-\frac{\partial g}{\partial u^{n-1}} d x^{n-1}\right\}
\end{aligned}
$$


and the vanishing of the terms in $d u^{i} \wedge d u^{n}$ required by (2.15) forces $\partial^{2} g / \partial u^{i} \partial u^{j}=0,1 \leq i, j \leq n-1$, so $g=f(x)+\sum g_{i}(x) u^{i}$, where $f \neq 0$. It is clear that this condition is also sufficient for $H=0$. When $H=0$ if follows from (2.14) that

$$
\eta^{1}=\frac{1}{f(x)}\left(d x^{n}-g_{1}(x) d x^{1}-\cdots-g_{n-1}(x) d x^{n-1}\right)
$$

If we define $X_{n}:=f(x) \partial / \partial x^{n}$ and $X_{i}:=\partial / \partial x^{i}+g_{i}(x) \partial / \partial x^{n}$ the the rank condition $[\mathbf{1}]$, [11] is satisfied unless $\left[X_{n}, X_{i}\right]=\left[X_{i}, X_{j}\right]=0$ for $1 \leq i, j \leq n-1$ and it follows directly from $\left(2.14^{\prime}\right)$ that this happens if and only if $d \eta^{1}=0$.

Next we proceed to the case in which $H$ has full rank. The infinitesimal action on $H$ is given by $d H-{ }^{\mathrm{t}} \beta H-H \beta \equiv 0\left(\bmod \eta^{1}, \bar{\eta}, \mu\right)$ which shows that $H$ is being conjugated. On an open set on which the rank and signature of $H$ are constant, we may normalize $H$ to a constant matrix $Q$ with the same rank and signature. The remaining torsion terms $S$ and $v$ may both be normalized to 0 , putting $d \eta^{1}$ in normal form:

$$
d \eta^{1}={ }^{\mathrm{t}} \mu Q \wedge \bar{\eta}
$$

As described in [4] and more recently in [5], the Euler-Lagrange equations for the functional $\int \eta^{1}$ are the exterior equations

$$
\begin{aligned}
& \mu=0 \\
& \bar{\eta}=0 .
\end{aligned}
$$

Theorem 2.3. If $\operatorname{rank} M=n-1$ and rank $H=n-1$ the system (2.17) yields closed loop time critical controls for (1.1).

Proof: The system $\left\{\eta^{2}, \ldots, \eta^{n}, \mu^{1}, \ldots, \mu^{n-1}\right\}$ is completely integrable and hence

$$
\mu \equiv T d w \quad\left(\bmod \eta^{2}, \ldots, \eta^{n}\right)
$$

for some non-singular matrix $T$ and vector function $w(x, u)$. Because

$$
0 \neq \mu^{1} \wedge \cdots \wedge \mu^{n-1} \wedge \eta^{1} \wedge \cdots \wedge \eta^{n}=\operatorname{det} T \operatorname{det} \frac{\partial w}{\partial u} d u^{1} \wedge \cdots \wedge d u^{n-1} \wedge \eta^{1} \wedge \cdots \wedge \eta^{n}
$$

the system $w=z, z$ constant, can be solved for $u^{i}(x) 1 \leq i \leq n-1$. But, as we have already observed, the solutions of $\frac{d x}{d t}=f(x, u(x))$ solve $\bar{\eta}=0$ and, by construction, also satisfy $\mu=0$. Thus they are solutions of the Euler-Lagrange system (2.17) and as such are time critical.

We also note that the same arguments given by Wilkens [13] show that there is a class of control systems for which the quadratic form $\left(\eta^{1}\right)^{2}+{ }^{\mathrm{t}} \bar{\eta} Q \bar{\eta}$ defines a pseudo-Riemannian metric on the state space and the solutions of (2.17) are geodesics of the metric.

Case 2) Rank $M=n-2$.

We conclude by considering the second class of problems. After the reduction of $\mathcal{M}$ the only unabsorbable torsion is in $d \eta^{n}$ and

$$
d \eta^{n}=\beta_{n} \wedge \eta^{n}+{ }^{\mathrm{t}} \bar{\mu} S \wedge \bar{\eta}+\left(\eta^{1},{ }^{\mathrm{t}} \bar{\eta}\right) T \wedge\left(\begin{array}{c}
\eta^{1} \\
\bar{\eta}
\end{array}\right)
$$

where ${ }^{\mathrm{t}} S=S$ and ${ }^{\mathrm{t}} T=-T$. 
TheOREM 2.4. If $\hat{g}=0$ then $d \eta^{n}$ can be put in the form (2.18) and $S=0$ if and only if the system is affine linear. If $S=0$ the system satisfies the rank condition for local controllability if and only if $T \neq 0$.

Proof: We can make the following choices for the 1 -forms $\eta_{U}, \mu_{U}$ :

$$
\begin{aligned}
& \eta_{U}^{1}=\frac{d x^{1}}{u^{1}} \\
& \bar{\eta}_{U}^{\alpha}=d x^{\alpha}-u^{\alpha} \frac{d x^{1}}{u^{1}} \quad 2 \leq \alpha \leq n-1 \\
& \eta_{U}^{n}=d x^{n}-\sum \frac{\partial g}{\partial u^{i}} d x^{i} \\
& \mu_{U}^{1}=\frac{d u^{1}}{u^{1}} \\
& \bar{\mu}_{U}^{\alpha}=d u^{\alpha}-u^{\alpha} \frac{d u^{1}}{u^{1}} \quad 2 \leq \alpha \leq n-1
\end{aligned}
$$

and the reduction of $\mathcal{M}$ imposes the following relations on the group of matricies defined by equation $\left(2.3^{\prime}\right)$

$$
\begin{aligned}
B & =\left(\begin{array}{cc}
B_{1} & B_{2} \\
0 & B_{3}
\end{array}\right) \quad B_{3} \in \mathbf{R} \\
E & =\left(\begin{array}{cc}
1 & A \\
0 & B_{1}
\end{array}\right) .
\end{aligned}
$$

Since

$$
\eta^{n}=B_{3}\left(d x^{n}-\sum \frac{\partial g}{\partial u^{i}} d x^{i}\right)
$$

It is clear from (2.18) that $S=0$ if and only if

$$
\frac{\partial^{2} g}{\partial u^{\alpha} \partial u^{\beta}}=0 \quad 2 \leq \alpha, \beta \leq n-1 .
$$

This condition together with the fact that

$$
\sum_{i=1}^{n-1} u^{i} \frac{\partial g}{\partial u^{i}}=g
$$

implies that $S=0$ if and only if

$$
\frac{\partial^{2} g}{\partial u^{i} \partial u^{j}}=0 \quad 1 \leq i, j \leq n-1
$$

If $S=0$ then

$$
\eta^{n}=B_{3}\left(d x^{n}-g_{1}(x) d x^{1}-\cdots-g_{n-1}(x) d x^{n-1}\right)
$$

and the condition $d \eta^{n} \equiv 0\left(\bmod \eta^{n}\right)$ is precisely the condition that the vector fields $X_{i}:=\partial / \partial x^{i}+$ $g_{i}(x) \partial / \partial x^{n}$ all commute.

\section{REFERENCES}

1. Boothby, W.M., Dayawansa, W.P., Some Global Aspects of the Feedback Linearization Problem, in "Differential Geometry: The Interface between Pure and Applied Mathematics," M. Lucksic, C. Martin, W. Shadwick eds. AMS Contemporary Mathematics 68, pp. 51-64.

2. Bryant, R., On notions of equivalence of variational problems with one independent variable, in "Differential Geometry: The Interface between Pure and Applied Mathematics," M. Lucksic, C. Martin, W. Shadwick eds. AMS Contemporary Mathematics 68, 1987, pp. 65-76. 
3. Cartan, E., Les sous-groupes des groupes continus de transformations, Euvres Complètes II, Ann. Ec. Normale 25 (1908), 719-856.

4. Cartan, E., "Leçons sur les Invariants Intégraux," Hermann, Paris, 1922.

5. Gardner, R. B., Differential Geometric methods interfacing control theory, in "Differential Geometric Control Theory," R. Brockett, R. Millman, H. Sussman eds., Progress in Mathematics 27, Birkhauser, Boston, MA, 1983, pp. 117-180.

6. Gardner, R. B., "NSF-CBMS Lectures on the Method of Equivalence with Applications to Control Theory" (to appear).

7. Gardner, R. B., Shadwick, W. F., Overdetermined Equivalence Problems with an Application to Feedback Equivalence, in "Differential Geometry: The Interface between Pure and Applied Mathematics," M. Lucksic, C. Martin, W. Shadwick eds. AMS Contemporary Mathematics 68, 1987, pp. 111-119.

8. Griffiths, P., "Exterior differential systems and the calculus of variations," Progress in Mathematics, Birkhauser, Boston, MA, 1983.

9. Hermann, R., The Theory of Equivalence of Pfaffian Systems and Input Systems Under Feedback, Math. Systems Theory 15 (1982), 343-356.

10. Hermes, H., Lasalle, J.P., Functional analysis and optimal control, Mathematics in Science and Engineering, section 2256 (1969), Academic Press, New Youk, London.

11. Krener, A., Normal Forms for Linear and Nonlinear Systems, in "Differential Geometry: The Interface between Pure and Applied Mathematics," M. Lucksic, C. Martin, W. Shadwick eds. AMS Contemporary Mathematics 68, 1987, pp. 157-189.

12. Sutton, M., Equivalence of Particle Lagrangians under contact Transformations, Ph.D. Dissertation, Mathematics Department, University of North Carolina, 1988..

13. Wilkens, G., Local feedback equivalence of control systems with 3 state and 2 control variables, Ph.D. Dissertation, Mathematics Department, University of North Carolina.

Keywords. Feedback equivalence of control systems, classical Lagrangian variational problems, time critical closed loop controls. 